\title{
Short-Term Effects of Strengthening Exercises of the Lower Limb Rehabilitation Protocol on Pain, Stiffness, Physical Function, and Body Mass Index among Knee Osteoarthritis Participants Who Were Overweight or Obese: A Clinical Trial
}

\author{
Muhammad Tariq Rafiq $\mathbb{D}^{1,2}$ Mohamad Shariff A. Hamid, ${ }^{3}$ and Eliza Hafiz \\ ${ }^{1}$ Centre for Sport and Exercise Sciences, University of Malaya, 50603 Kuala Lumpur, Malaysia \\ ${ }^{2}$ Rehmatul-Lil-Alameen Postgraduate Institute of Cardiology, Punjab Employees Social Security Institution, Lahore, Pakistan \\ ${ }^{3}$ Unit of Sports Medicine, Faculty of Medicine, University of Malaya, 50603 Kuala Lumpur, Malaysia \\ Correspondence should be addressed to Muhammad Tariq Rafiq; drtariqrafiq149@yahoo.com
}

Received 24 May 2021; Revised 2 November 2021; Accepted 9 November 2021; Published 22 December 2021

Academic Editor: Noureddin Nakhostin Ansari

Copyright $(02021$ Muhammad Tariq Rafiq et al. This is an open access article distributed under the Creative Commons Attribution License, which permits unrestricted use, distribution, and reproduction in any medium, provided the original work is properly cited.

\begin{abstract}
Background. Osteoarthritis (OA) of the knee is defined as a progressive disease of the synovial joints and is characterized by wear and tear of the cartilage and underlying bone. This study aimed to determine the short-term effects of the lower limb rehabilitation protocol (LLRP) on pain, stiffness, physical function, and body mass index (BMI) among knee OA participants who were overweight or obese. Methodology. A single-blinded randomized controlled trial of one-month duration was conducted at Rehmatul-Lil-Alameen Postgraduate Institute, Lahore, Pakistan. Fifty overweight or obese participants with knee OA were randomly divided into two groups by a computer-generated number. Participants in the rehabilitation protocol group (RPG) were provided with leaflets explaining the strengthening exercises of the LLRP and instruction of daily care (IDC), while the participants in the control group (CG) were provided with leaflets explaining the IDC only for a duration of four weeks. The primary outcome measures were the Western Ontario and McMaster Universities Osteoarthritis Index (WOMAC) scores for pain, stiffness, and physical function. The secondary outcome measures were BMI, exercise adherence, and patients' satisfaction assessed by using the numeric rating scale ranging from 0 to 10 . The paired-sample $t$-test was used to analyze the differences within groups from baseline to posttest evaluations. The analysis of variance $2 \times 2$ factor was used to analyze the differences in BMI, knee pain, stiffness, and physical function between the groups. Results. Participants in the RPG and CG reported a statistically significant reduction in knee pain and stiffness $(p \leq 0.05)$ within the group. The reduction in the scores of knee pain was higher in participants in the RPG than that in participants in the CG $(p=0.001)$. Additionally, participants in the RPG reported greater satisfaction $(p=0.001)$ and higher self-reported exercise adherence $(p=0.010)$ and coordinator-reported exercise adherence $(p=0.046)$ than the participants in the CG. Conclusion. Short-term effects of the LLRP appear to reduce knee pain and stiffness only, but not physical function and BMI.
\end{abstract}

\section{Introduction}

The knee joint is a complex synovial joint in the human body where the femur, tibia, fibula, and patella articulate [1]. Articulation is supported by structures that include the muscles, ligaments, tendons, articular cartilage, synovial membrane, synovial capsule, meniscus, and fat pad [2]. The synovial fluid and articular cartilage lubricate the knee allowing low-friction joint movement [3]. The articular cartilage protects the subchondral bone from local stresses because of its strong reported excessive joint load in knee osteoarthritis (OA) [4]. A study provides evidence on the fact that excessive joint load in knee osteoarthritis (OA) patients can lead to an increased inflammatory response, 
joint pain, and swelling [5]. A recent study compared the body mass index (BMI) of obese and nonobese knee OA elderly individuals and reported that obesity resulted in less functional mobility, slower gait speed, higher pain intensity, and difficulty in performing daily living activities than nonobese individuals [6]. It was reported that obesity increases the joint load in knee OA patients, resulting in lowering functional mobility and increasing pain intensity. Over the past decade, the prevalence rate of overweight and obesity has increased in the United States of America (USA), Canada, Mexico, France, and Switzerland [7]. However, the prevalence of obesity solely among men and women of the USA population was 37.9 and $41.1 \%$, respectively [8]. A recent retrospective study investigated the physical and functional characteristics of 320 patients with knee OA, reporting that obesity and advanced age were associated with an increased risk of knee OA [9]. Yet another study in the perspective under discussion reached the conclusion that overweight and obesity had a negative impact on increasing pain perception among patients with OA [10].

A study suggested that the reduced weight may be related to less demand on the proximal muscles and the internal medial rotator muscle of the knee, to provide stability at the toe-off during excessive rearfoot motion [11]. A recent exposition led to the fact that by acquiring the habit of regular exercise, knee $\mathrm{OA}$ patients can reduce pain and improve the quality of life and physical activity. One of the defense mechanisms of the knee, such as body weight control, is useful for healthy aging [9]. All international clinical practice guidelines recommend patients with knee $\mathrm{OA}$, who are overweight or obese, to lose their weight. Adherence may refer to different things and can be used to evaluate the attendance, technique, or accuracy of exercise protocols in supervised appointments [12]. Research in the context of adherence refers to the accomplishment of selfreported and coordinator-reported adherence by the intervention groups.

Clinical guidelines recommend exercise therapy as the primary nonpharmacologic treatment for knee OA [13]. Because of remarkable evidence demonstrating the beneficial effects of physical exercise among patients with OA, exercise is often indicated as one of the main components in the rehabilitation process $[14,15]$. Among the several types of physical exercise programs, muscle strengthening is important because of the relationship between muscle weakness, pain, and malfunction [16, 17]. A current systematic review on nonpharmacological interventions for treating symptoms of knee $\mathrm{OA}$ in overweight or obese patients resulted that the most effective intervention that showed improvement of knee pain and function was strengthening exercise. Similarly, it also reported that the combination of diet and exercise was found effective in reducing weight and improving knee pain [18]. A study explained that progressive resistance strength training increases the load gradually over the training course to strengthen the major muscle groups and has been recommended to prevent or reduce late-life disability for older adults [19]. The effectiveness of rehabilitation in non-weightbearing positions as well as strengthening exercises of major muscle groups of the lower limbs may provide more objective data than the standard rehabilitation approaches we are using today to treat overweight and obese knee OA patients. However, there is a gap in knowledge regarding whether strengthening exercises of major muscle groups of lower limbs in non-weight-bearing positions can improve the effects of rehabilitation among overweight or obese knee OA participants. Hence, the current randomized controlled trial aimed to determine the short-term effects of strengthening exercise of the lower limb rehabilitation protocol (LLRP) in non-weight-bearing positions on knee pain, stiffness, physical function, BMI, patients' satisfaction, and exercise adherence in overweight or obese knee OA participants.

The current study aimed to determine the short-term effects of strengthening exercises of LLRP in non-weightbearing positions on pain, stiffness, physical function, BMI, patients' satisfaction, and exercise adherence in overweight or obese knee OA participants.

\section{Methodology}

2.1. Design and Setting. This is a single-blinded randomized controlled trial that involved participants diagnosed with knee OA who are overweight or obese. Participants were randomized into the rehabilitation protocol group (RPG) and the control group (CG) using a computer-generated simple randomization technique. The study was conducted at the teaching bay of Rehmatul-Lil-Alameen Postgraduate Institute of Cardiology (RAIC), Punjab Employees Social Security Institution (PESSI), Lahore, Pakistan. All participants were asked to complete the clinical research form (CRF) following randomization. The CRF gathered sociodemographic information, symptoms of knee pain and stiffness, physical function scores, and BMI. The participants' satisfaction and exercise adherence were collected after four weeks of intervention.

Participants in each treatment group were provided with necessary details about their intervention protocol after randomization. Making explanation of the purpose and constraints of the study, the participants were asked to provide written informed consent for their participation in the study. All participants were also given a diary and asked to record the attendance of completion of their interventions based on leaflets. The current study was approved by the ethical committee of Rehmatul-Lil-Alameen Postgraduate Institute of Cardiology, Punjab Employees Social Security Institution, with reference number RAIC PESSI/Estt/2020/36 on 20-052020, and the trial was registered in the Iranian Registry of Clinical Trials with reference number https://trialsearch.who. int/?TrialID=IRCT20191221045846N2 on 28-06-2020.

\subsection{Study Participants, Recruitment, and Selection.} Participants with knee OA who were overweight or obese from the urban community of Punjab, Lahore, Pakistan, were screened. The sample included males and females with OA on one or both knees as confirmed by a medical specialist according to the Kellgren and Lawrence radiographic 
scale for the assessment of OA [20]. Plain radiography was performed to obtain anteroposterior and lateral views of the affected knee/knees in the standing position at the AlRehmat Trust Hospital, Lahore.

Participant inclusion criteria were as follows:

(i) Aged between 45 and 60 years

(ii) Having minimum qualification of matriculation

(iii) History of knee pain for more than three months

(iv) Overweight $\quad\left(\mathrm{BMI} \geq 25 \mathrm{~kg} / \mathrm{m}^{2}\right) \quad$ or obese $\left(\mathrm{BMI}>30 \mathrm{~kg} / \mathrm{m}^{2}\right)[21]$

(v) Diagnosed with mild or moderate knee OA according to the Kellgren and Lawrence radiographic scale [20]

Participant exclusion criteria were as follows:

(i) Diagnosed with rheumatoid arthritis, systemic lupus erythematosus, flat foot/feet, or spinal deformities

(ii) History of metabolic, hormonal, orthopaedic, or cardiovascular disease

(iii) Previous surgery of the knee/s [22]

(iv) Inability to walk independently

(v) Injection of knee/s for the last six months [22]

All information related to inclusion and exclusion criteria was gathered from the predefined questionnaire.The researcher recruited the participants by using active recruitment strategies such as urban political and welfare organizations via word of mouth by the convenience sampling technique. The list of participants with knee OA in the studied area was obtained from the welfare organization by explaining the benefits of study participation. Two study coordinators prepared the list of potential participants with knee OA in the recruitment area. After obtaining the list of potential participants with knee OA, the researcher arranged a meeting with the knee OA participants by calling them on the phone. The meeting was held at the teaching bay of RAIC, PESSI, Lahore, Pakistan, in the presence of a medical specialist. Participants were screened for eligibility to participate in the study. Only participants fulfilling the inclusion criteria of the study were invited to participate in this study.

2.3. Sample Size. Sample size estimation was performed using the $\mathrm{G} *$ Power 3.1 .3 software. By assuming the medium effect size $f=0.70$ and setting $\alpha=0.05$, power $(1-\mathrm{B})=0.80$, number of groups $=2$, and number of measurements $=2$, the total sample size estimated was 33 participants. After considering the apprehension of dropout or mortality during the research period, the sample size of 50 participants for the two groups was decided.

2.4. Blinding and Allocation. The principle investigator was not blinded in the study. The participants receiving the intervention were kept blinded by simply not informing them of their treatment allocation. The coordinators collecting data were independent individuals from the trials and were unaware of the group allocation. There were different coordinators at the baseline and posttest evaluation. Individuals performing the statistical analysis were kept blinded by labelling the groups with nonidentifying terms (such as $X$ and $Y$ ).

2.5. Study Randomization. After completing the screening of knee OA participants, the researcher allocated 50 selected participants into two groups, namely, RPG and CG, by using a computer-generated number. Each group consisted of 25 participants. The participants receiving the intervention were blinded by their treatment allocation. The participants in the RPG followed the strengthening exercise of the LLRP and followed the instructions of daily care (IDC) for a duration of 4 weeks. The participants in the CG were not involved in the rehabilitation protocols, but these participants only followed the IDC for a duration of 4 weeks.

\subsection{Research Procedures}

2.6.1. Research Procedure of the RPG. The researcher taught the strengthening exercises of the LLRP and IDC to the RPG for a duration of four weeks. Participants were advised to continue performing the strengthening exercises of the LLRP three times a week for four weeks at home. These training sessions included strengthening exercises for the lower limbs in non-weight-bearing, sitting, or lying positions. Each training session started with 10 minutes of warm-up, 45-60 minutes of lower limb resistance training, and 10 minutes of cooldown at the end of the training protocol.A cooldown period is essential after a training session and should last approximately 5-10 minutes $[23,24]$. When static stretching is used as a part of warm-up immediately prior to exercise, it causes harm to muscle strength [25]. The participants in the RPG performed the strengthening exercises of the LLRP and followed the IDC at home for four weeks. The contents of the IDC are explained in a recent randomized controlled trial [22].

The sequence of the training program started with 10 minutes of warm-up with whole body range of motion $(\mathrm{ROM})$ and dynamic stretching exercises (Table 1). The participants performed 10 repetitions of ROM exercises for each muscle group and five repetitions of dynamic stretching exercises for each muscle group as a part of warm-up. After the warm-up, the participants performed the strengthening exercises of the LLRP for the stipulated weeks as stated in Table 2. After completing the strengthening exercises, the participants performed the 10-minute cooldown with whole body ROM and static stretching exercises (Table 1). The participants performed 10 repetitions of ROM exercises for each muscle group and three repetitions of static stretching exercises for each muscle group as a part of cooldown.

2.6.2. Research Procedure of the CG. The participants in the CG were asked to follow the IDC only for a duration of four weeks. The IDC were also translated into the Urdu language by two language experts as the participants preferred the 
TABLE 1: Whole body ROM exercises as part of warm-up or cooldown.

\begin{tabular}{|c|c|}
\hline Body part & ROM exercises \\
\hline Neck & Flexion and extension, side flexion to the right and then to the left, and neck rotation to the right and then to the left \\
\hline Shoulder & Shoulder flexion and extension, shoulder abduction and adduction, and shoulder rotation \\
\hline Elbow & Elbow flexion and extension \\
\hline Wrist & Wrist flexion and extension \\
\hline Spine & Spine flexion and extension and spine rotation to the right and then to the left \\
\hline Hip & Hip abduction and adduction and hip flexion and extension \\
\hline Knee & Knee flexion and extension \\
\hline Ankle & Ankle dorsiflexion and plantar flexion \\
\hline 10 repe & of each muscle group's ROM exercises will be performed before and after the inter \\
\hline
\end{tabular}

ROM, range of motion.

TABLE 2: Strengthening exercises of the LLRP in non-weight-bearing sitting and lying positions.

\begin{tabular}{lcccc}
\hline Muscle group & Position & Resistance & For two weeks $\left(1^{\text {st }}\right.$ and $2^{\text {nd }}$ week $)$ & For two weeks $\left(3^{\text {rd }}\right.$ and $4^{\text {th }}$ week $)$ \\
\hline Hip abductors & Supine lying & Resistance band & 2 sets of 7 reps & 2 sets of 10 reps \\
Hip adductors & Supine lying & Resistance band & 2 sets of 7 reps & 2 sets of 10 reps \\
Hip flexors & Side lying & Resistance band & 2 sets of 7 reps & 2 sets of 10 reps \\
Hip extensors & Side lying & Resistance band & 2 sets of 7 reps & 2 sets of 10 reps \\
Quadriceps (knee) & Side lying & Ankle weight & 2 sets of 7 reps & 2 sets of 10 reps \\
Hamstrings (knee) & Side lying & Ankle weight & 2 sets of 7 reps & 2 sets of 10 reps \\
Ankle dorsiflexors & Side lying & Foot weight & 2 sets of 7 reps & 2 sets of 10 reps \\
Ankle plantar flexors & Side lying & Foot weight & 2 sets of 7 reps & 2 sets of 10 reps \\
\hline
\end{tabular}

LLRP, lower limb rehabilitation protocol; reps, repetitions.

Urdu translation for better understanding based on a recent pilot study [26].

2.7. Outcome Measures. The outcome measures were collected at baseline and after 4 weeks of intervention. Outcome measures were categorized into primary and secondary outcome measures.

2.7.1. Primary Outcome Measures. These were knee pain, stiffness, and physical function assessed using the Western Ontario and McMaster Universities Osteoarthritis Index (WOMAC) score. The WOMAC score is widely accepted and validated [27]. The WOMAC score ranges from 0 to 4 on a Likert-type scale; the higher the score, the worse the pain, stiffness, and physical function.

2.7.2. Secondary Outcome Measures. These were BMI, participant's satisfaction, and exercise adherence. The BMI was calculated using the formula (weight (kilogram)/height ${ }^{2}$ (meter squared). Both the participants' satisfaction and participants' adherence to the interventions were assessed using a numeric rating scale ranging from 0 to 10 .

Participants' satisfaction with the RPG or CG intervention was determined by asking "How satisfied have you been with your leaflet-provided intervention exercise program at home over the past 4 weeks?" The answers on the rating scale ranged from zero= "not at all satisfied" to $10=$ "extremely satisfied." The numeric rating scale of a published study in which the participants were instructed to rate their satisfaction ranging from $0=$ "not at all satisfied" to
$10=$ "extremely satisfied" was used [28]. Responses to the two interventions were analyzed separately.

Self-reported exercise adherence was measured by using a numerical scale ranging from zero=never performed intervention of the RPG or CG to $10=$ always performed intervention of the RPG or CG. Numerical rating scales from 0 to 10 have good validity and reliability and have also been widely used in other trials [29, 30].

A study coordinator, who was blinded to the participant's intervention, contacted all participants through a phone call upon study completion. The participants were asked about an opinion regarding the intervention of RPG or CG adherence. The blinded coordinator provided a score in response to the question "what is the score of the 4-week RPG or CG intervention?" on a scale from zero= "never performed the intervention" to $10=$ "always performed the intervention" according to their provided leaflets.

2.8. Statistical Procedures. Statistical Package for Social Sciences, version 22, Chicago, IL, was used to manage and analyze the data. Descriptive statistics was used for the demographic questionnaire and for the mean and standard deviation of all variables. Inferential statistics was used for all quantitative measures. Prior to data analysis, the Shapiro-Wilk test was used for all variables to check the normality of data. The scores were normally distributed; therefore, the paired-sample $t$-test was used to analyze the differences of outcome measures within groups from baseline to posttest measurements. Analysis of variance $2 \times 2$ factor was used to compare the differences of clinical outcome measures between the groups. The independent-sample $t$-test was used to evaluate the mean (95\% confidence interval (CI)) difference 


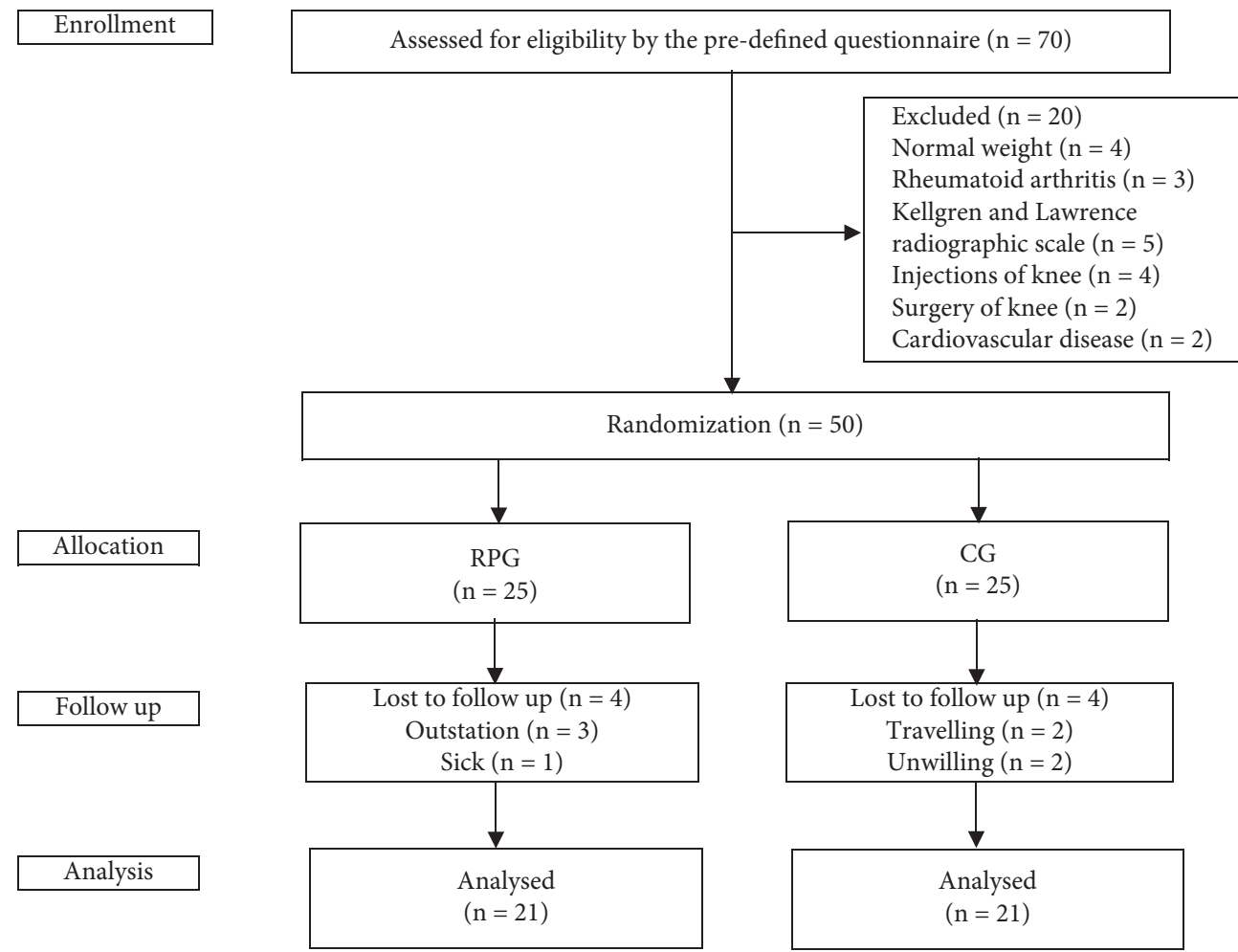

FIGURE 1: Flow chart of participants' participation; RPG, rehabilitation protocol group; CG, control group; and n, number.

TABLe 3: Baseline demographic characteristics and clinical outcome measures of participants at the baseline: mean (SD) or $n$ (\%).

\begin{tabular}{|c|c|c|c|c|}
\hline Baseline demographic characteristics and clinical outcome measures & Overall $(n=50)$ & RPG $(n=25)$ & CG $(n=25)$ & $p$ value \\
\hline Age, mean (SD), y & $53.12(5.41)$ & $53.40(5.18)$ & $52.84(5.74)$ & 0.719 \\
\hline Gender (male/female), $n$ & $23 / 27$ & $11 / 14$ & $12 / 13$ & 0.782 \\
\hline \multicolumn{5}{|l|}{ Educational status, $n$ (\%) } \\
\hline (i) Matriculation & $8(16)$ & $6(24.0)$ & $2(8.0)$ & 0.473 \\
\hline (ii) Intermediate & $20(40)$ & $8(32.0)$ & $12(48.0)$ & \\
\hline (iii) Bachelor & $13(26)$ & $7(28.0)$ & $6(24.0)$ & \\
\hline (iv) Master & $9(18)$ & $4(16.0)$ & $5(20.0)$ & \\
\hline \multicolumn{5}{|l|}{ Employment, no. (\%) } \\
\hline (i) Yes & $33(66)$ & $19(76.0)$ & $14(56.0)$ & 0.141 \\
\hline (ii) No & $17(34)$ & $6(24.0)$ & $11(44.0)$ & \\
\hline Weight (kg), mean (SD) & $84.06(11.09)$ & $86.68(8.15)$ & $81.44(13.05)$ & 0.095 \\
\hline Height (m), mean (SD) & $2.63(0.31)$ & $2.72(0.34)$ & $2.54(0.25)$ & 0.036 \\
\hline $\operatorname{BMI}\left(\mathrm{kg} / \mathrm{m}^{2}\right)$, mean $(\mathrm{SD})$ & $32.09(4.16)$ & $32.18(4.49)$ & $32.01(3.89)$ & 0.885 \\
\hline WOMAC pain $(0-20)$, mean $(S D)$ & $9.94(1.73)$ & $9.84(1.51)$ & $10.04(1.94)$ & 0.687 \\
\hline WOMAC stiffness $(0-8)$, mean $(S D)$ & $3.70(1.70)$ & $3.84(1.90)$ & $3.56(1.50)$ & 0.567 \\
\hline WOMAC physical function $(0-68)$, mean (SD) & $23.60(12.14)$ & $24.48(12.77)$ & $22.72(11.68)$ & 0.614 \\
\hline
\end{tabular}

RPG, rehabilitation protocol group; CG, control group; SD, standard deviation; no., number; kg, kilogram; m, meter.

between groups for exercise adherence and participants' satisfaction measured after 4 weeks of intervention.

\section{Results}

A total of seventy participants were screened and assessed for eligibility for inclusion in this study. Twenty participants were excluded for reasons as shown in Figure 1; the remaining fifty participants were randomized into the RPG or CG. Of the twenty-five participants allocated to the RPG, 4 participants did not continue with their intervention because they were outstation due to occupation and sick. Likewise, 4 of the twenty-five participants allocated to the CG did not continue the intervention because they were travelling and unwilling. We could not obtain the postintervention outcomes for these 8 withdrawn participants. A final total of forty-two participants (twenty-one in the RPG and twenty-one in the CG) completed the study and the data of which were analyzed (Figure 1).

Demographic characteristics and clinical outcome measures of the study participants at baseline are shown in Table 3. No significant differences were observed in baseline 
TABLE 4: Clinical outcome measures of study participants within groups.

\begin{tabular}{lcccc}
\hline Clinical outcome measures & Baseline evaluation, mean (SD) & Posttest evaluation, mean (SD) & Mean difference (95\% CI) & $p$ value \\
\hline Rehabilitation protocol group (RPG) $(n=21)$ & & & \\
BMI, kg/m & & $31.87(4.33)$ & $-1.10(-3.58$ to 1.37$)$ & 0.364 \\
WOMAC pain $(0-20)$ & $9.71(1.58)$ & $8.85(1.49)$ & $0.85(0.44$ to 1.27$)$ & $\leq \mathbf{0 . 0 0 1}$ \\
WOMAC stiffness (0-8) & $3.76(1.84)$ & $3.04(1.49)$ & $0.71(0.50$ to 0.92$)$ & $\leq \mathbf{0 . 0 0 1}$ \\
WOMAC physical function (0-68) & $24.09(12.75)$ & $23.90(12.37)$ & $0.19(-0.04$ to 0.42$)$ & 0.104 \\
\hline Control group $(C G)(n=21)$ & & & \\
BMI, kg/m & $31.76(3.66)$ & $31.67(3.59)$ & $0.08(-0.03$ to 0.20$)$ \\
WOMAC pain (0-20) & $9.76(1.97)$ & $9.52(2.08)$ & 0.159 \\
WOMAC stiffness (0-8) & $3.42(1.56)$ & $3.21(1.41)$ & $0.23(-0.01$ to 0.49$)$ & 0.066 \\
WOMAC physical function (0-68) & $22.19(11.06)$ & $22.07(11.00)$ & $0.21(-0.03$ to 0.45$)$ & 0.083 \\
\hline
\end{tabular}

RPG, rehabilitation protocol group; CG, control group; SD, standard deviation; $n$, number; BMI, body mass index; WOMAC, Western Ontario and McMaster Universities Osteoarthritis Index; CI, confidence interval; SD, standard deviation.

demographic characteristics and clinical outcome measures of pain, stiffness, physical function, and BMI between the 2 groups $(p>0.05)$.

Table 4 shows that, after 4 weeks of intervention, the participants in the RPG reported a significant reduction in pain $(p \leq 0.001)$ and stiffness $(p \leq 0.001)$, but no improvement in physical function $(p=0.104)$ and BMI $(p=0.364)$ within the group. The participants in the CG also reported a reduction in pain, knee stiffness, physical function, and BMI scores in week 4 compared to baseline; the differences, however, were not statistically significant $(p>0.05)$ within the group. Mean values and $95 \% \mathrm{CI}$ of outcome measures within groups are shown in Figure 2.

When the effectiveness of outcome measures was compared between the 2 groups, the participants in the RPG reported a statistically more significant improvement in the WOMAC pain $(p=0.016)$ and stiffness score $(p=0.002)$ than those in the CG. However, the participants in the RPG reported no statistically significant improvement in the scores of BMI and WOMAC physical function $(p>0.05)$ than those in the CG (Table 5).

The mean between-group difference for participants' satisfaction was 1.95 (95\% CI 0.91 to 2.98 ) with a $p$ value of 0.001 in favour of the RPG. There was a statistically significant between-group difference for self-reported exercise adherence, with a mean between-group difference of 1.33 (95\% CI 0.34 to 2.32 ) with a $p$ value of 0.010 in favour of the RPG. Similarly, there was a statistically significant betweengroup difference for coordinator-reported exercise adherence, with a mean between-group difference of 0.88 (95\% CI 0.01 to 1.75 ) with a $p$ value of 0.046 in favour of the RPG (Table 6).

There were no adverse events as well as suspected unexpected serious adverse reactions reported in the current study.

\section{Discussion}

To the best of our knowledge, this is the first randomized controlled trial to address the short-term effects of strengthening exercises of the LLRP in improving pain, stiffness, physical function, BMI, patients' satisfaction, and exercise adherence among knee OA participants who are overweight or obese. Our results indicate that including the short-term effects of strengthening exercises of the LLRP could improve pain and stiffness more efficiently than usual care could. Similarly, the results of this study indicate that participants in the RPG reported greater satisfaction and adherence to their intervention compared to the participants in the CG.

The results for reducing pain of the current study are consistent with an overview of nine systematic reviews [31] and a recent randomized controlled trial [32] that reported that exercise interventions for knee OA reduce pain but the effect sizes are considered small. In the current study, the strengthening exercises of the LLRP were performed in nonweight-bearing positions without putting mechanical pressure on the knee. Therefore, instead of a small duration, it reported a significant reduction in pain and stiffness. An overview of nine systematic reviews [31] and a randomized controlled trial [32] also reported significant improvement in physical function, but the current study's results reported no improvement in physical function. It may be due to the small duration of the current study. The results of weight loss of the current study are inconsistent with a pragmatic randomized controlled trial that reported that telephonebased weight loss support did not affect weight [33]. A recent study concluded that the progressive resistance strength training of the LLRP in non-weight-bearing positions in patients with knee OA is effective in reducing BMI [34].

A published study of a short duration of 24 hours contradicts the current study and reported that moderate strengthening exercises did not have an effect on knee pain, but only induce a mild inflammatory response [35]. A study reported that an 18-month combined exercise and dietary weight-loss intervention of 316 overweight or obese individuals with radiographic evidence of knee OA was effective in improving knee pain as well as physical function [36]. It supports the current study's results of knee pain. The results of a randomized controlled trial in which $90 \%$ participants were satisfied with their intervention of home exercise programs provided with an app with remote support [37] are the same as those of the current study. However, participants who received their intervention from home exercise programs with an app with remote support reported greater adherence [35] than those in the current study. This current 

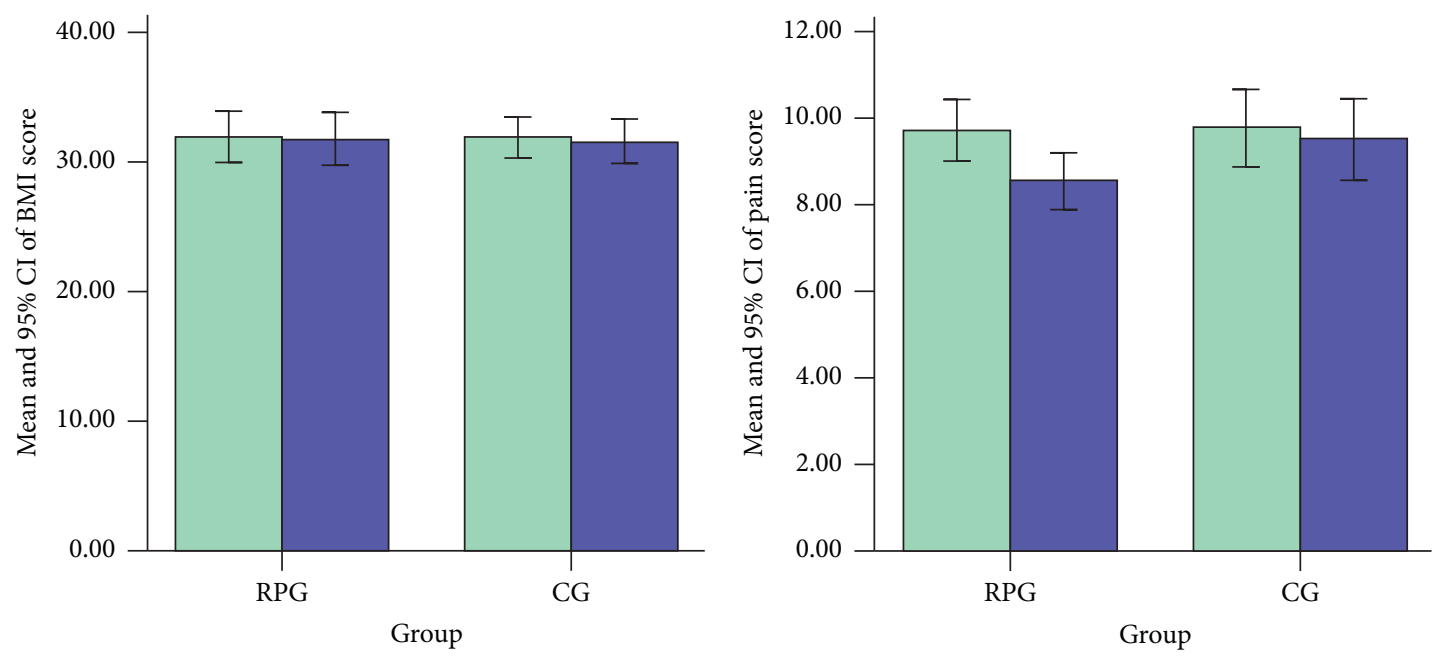

Outcome measures:

Outcome measures:

$\square$ Baseline

$\square$ Baseline

Post-test

(a)

(b)

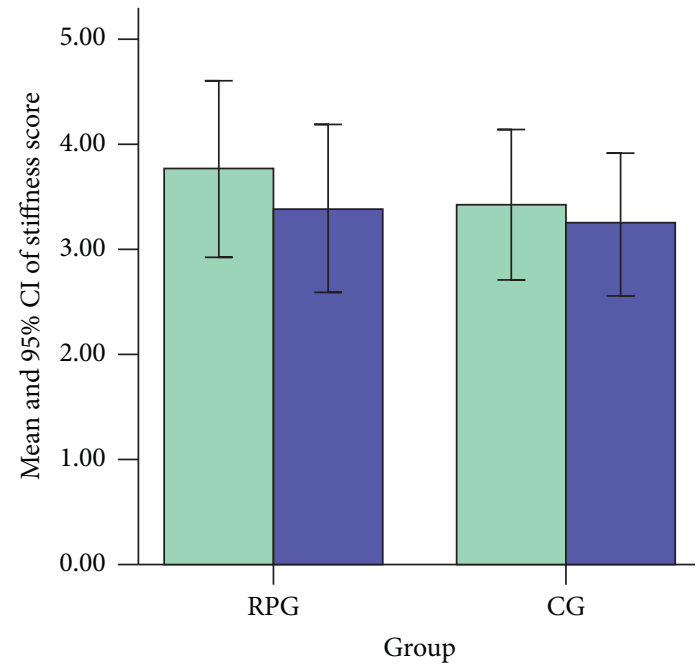

Outcome measures

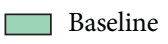

Post-test

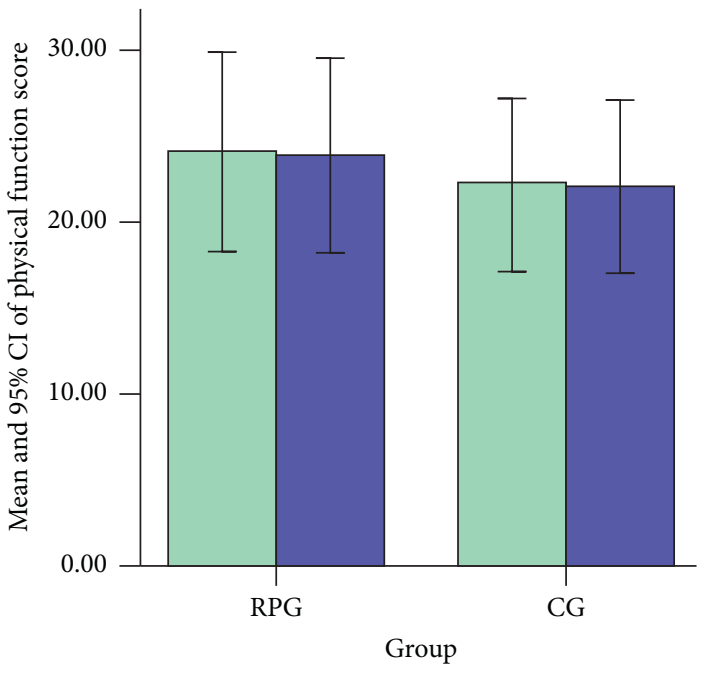

Outcome measures:

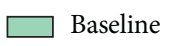

Post-test

(c)

(d)

Figure 2: Mean and 95\% CI of the outcomes measures within groups. (a) Mean and 95\% CI of BMI score at baseline and 3-month follow-up, (b) mean and 95\% CI of pain score at baseline and 3-month follow-up, (c) mean and 95\% CI of stiffness score at baseline and 3-month follow-up, and (d) mean and 95\% CI of physical function score at baseline and 3-month follow-up; RPG, rehabilitation protocol group; CG, control group; BMI, body mass index; and CI, confidence interval.

TABLE 5: Comparison of clinical outcome measures between the groups (RPG and CG).

\begin{tabular}{lccc}
\hline Clinical outcome measures & RPG & CG & Mean difference (95\% CI) \\
& Mean difference $(95 \% \mathrm{CI})$ & $0.08(-0.03$ to 0.20$)$ & \\
\hline BMI, $\mathrm{kg} / \mathrm{m}^{2}$ & $-1.10(-3.58$ to 1.37$)$ & $0.23(-0.01$ to 0.49$)$ & 0.868 \\
WOMAC pain $(0-20)$ & $0.85(0.44$ to 1.27$)$ & $0.21(-0.03$ to 0.45$)$ & $\mathbf{0 . 0 1 6}$ \\
WOMAC stiffness $(0-8)$ & $0.71(0.50$ to 0.92$)$ & $0.11(-0.05$ to 0.29$)$ & $\mathbf{0 . 0 0 2}$ \\
WOMAC physical function $(0-68)$ & $0.11(-0.05$ to 0.29$)$ & 0.576 \\
\hline
\end{tabular}

RPG, rehabilitation protocol group; CG, control group; BMI, body mass index; WOMAC, Western Ontario and McMaster Universities Osteoarthritis Index; CI, confidence interval. 
TABLE 6: Mean (SD) of groups and mean (95\% CI) difference between groups for exercise adherence and participants' satisfaction measured after 4 weeks of interventions at posttest evaluation.

\begin{tabular}{|c|c|c|c|c|}
\hline Outcomes & RPG & CG & RPG minus CG & $p$ value \\
\hline Self-reported exercise adherence (0 to 10$)$ & $7.22(1.47)$ & $5.88(1.45)$ & 1.33 (0.34 to 2.32$)$ & 0.010 \\
\hline Coordinator-reported exercise adherence ( 0 to 10$)$ & $7.02(1.31)$ & $6.13(1.25)$ & $0.88(0.01$ to 1.75$)$ & 0.046 \\
\hline Participants' satisfaction with the groups (0 to 10$)$ & $8.92(1.39)$ & $6.97(1.66)$ & $1.95(0.91$ to 2.98$)$ & $\leq \mathbf{0 . 0 0 1}$ \\
\hline
\end{tabular}

RPG, rehabilitation protocol group; CG, control group.

randomized controlled trial provides further evidence that the LLRP that includes training sessions of strengthening exercises in non-weight-bearing positions is more effective for overweight or obese knee OA participants than typical rehabilitation.

This study has several limitations. First, the results may not be generalized to all overweight or obese knee OA participants because we enrolled OA participants with a grading scale of 2- mild or 3- moderate according to the Kellgren and Lawrence radiographic scale. No long-term follow-up records were taken. Finally, comparisons were performed on a relatively smaller number of participants in this study. Thus, further research with a larger sample size and long-term follow-up is required to confirm the results of strengthening exercises of the LLRP.

\section{Conclusions}

The current study showed the advantage of strengthening exercises of the LLRP in non-weight-bearing positions on knee pain and stiffness reduction in overweight or obese participants with knee OA compared with the IDC without strengthening exercises. Therefore, strengthening exercises of the LLRP in non-weight-bearing positions may be an effective intervention to reduce knee pain and stiffness. The current study also showed that there was no improvement in physical function and BMI due to strengthening exercises of the LLRP that were performed for a duration of 4 weeks. In the management of overweight or obese participants with knee OA, strengthening exercises of major muscle groups of lower limbs in nonweight-bearing positions may reduce knee pain and stiffness and be an effective additional treatment option in the rehabilitation program.

\section{Data Availability}

The dataset of this manuscript is available from the corresponding author upon reasonable request.

\section{Disclosure}

A preprint was submitted at https://www.medrxiv.org/ content/10.1101/2021.11.20.21266300v1 [38].

\section{Conflicts of Interest}

The authors declare no conflicts of interest.

\section{Acknowledgments}

The authors are so grateful to all the participants in this study for their kindness and courage and for their valuable contribution to achieve the rehabilitation protocol.

\section{Supplementary Materials}

Additional File 1: trial protocol. Additional File 2: CONSORT 2010 checklist. Additional File 3: CONSORT 2010 flow diagram. (Supplementary Materials)

\section{References}

[1] M. D. Miller and S. R. Thompson, DeLee \& Drez's Orthopaedic Sports Medicine E-Book, Elsevier Health Sciences, Amsterdam. Netherlands, 2014.

[2] M. T. Hirschmann and W. Müller, "Complex function of the knee joint: the current understanding of the knee," Knee Surgery, Sports Traumatology, Arthroscopy, vol. 23, no. 10, pp. 2780-2788, 2015.

[3] M. Huber, S. Trattnig, and F. Lintner, "Anatomy, biochemistry, and physiology of articular cartilage," Investigative Radiology, vol. 35, no. 10, pp. 573-580, 2000.

[4] J. Podlipská, J. M. Koski, and E. Liukkonen, "Non-invasive semi-quantitative and quantitative ultrasonography findings in knee osteoarthritis: preliminary study," Osteoarthritis and Cartilage, vol. 22, pp. S278-S279, 2014.

[5] I. C. Helmark, U. R. Mikkelsen, J. Børglum et al., "Exercise increases interleukin-10 levels both intraarticularly and perisynovially in patients with knee osteoarthritis: a randomized controlled trial," Arthritis Research and Therapy, vol. 12, no. 4, p. R126, 2010.

[6] M. Gomes-Neto, A. D. Araujo, I. D. A. Junqueira, D. Oliveira, A. Brasileiro, and F. L. Arcanjo, "Comparative study of functional capacity and quality of life among obese and nonobese elderly people with knee osteoarthritis," Revista Brasileira de Reumatologia, vol. 56, no. 2, pp. 126-130, 2016.

[7] https://www.oecd.org/els/health-systems/Obesity-Update2017.pdf.

[8] C. M. Hales, M. D. Carroll, C. D. Fryar, and C. L. Ogden, Prevalence of Obesity Among Adults and Youth: United States, 2015-2016. NCHS Data Brief, No 288, National Center for Health Statistics, Hyattsville, MD, USA, 2017.

[9] S. Bakirhan, O. Bozan, B. Unver, and V. Karatosun, "Evaluation of functional characteristics in patients with knee osteoarthritis," Acta Ortopédica Brasileira, vol. 25, no. 6, pp. 248-252, 2017.

[10] F. M. Alfieri, N. C. d. O. V. e. Silva, and L. R. Battistella, "Study of the relation between body weight and functional limitations and pain in patients with knee osteoarthritis," Einstein (São Paulo), vol. 15, no. 3, pp. 307-312, 2017. 
[11] S. P. Messier, D. J. Gutekunst, C. Davis, and P. DeVita, "Weight loss reduces knee-joint loads in overweight and obese older adults with knee osteoarthritis," Arthritis \& Rheumatism, vol. 52, no. 7, pp. 2026-2032, 2005.

[12] L. Tara, "The effectiveness of an app with remote support to improve adherence to home exercise programs prescribed by physiotherapists: a randomised controlled trial," Master's Thesis, University of Sydney, Sydney, Australia, 2018.

[13] A. E. Nelson, K. D. Allen, Y. M. Golightly, A. P. Goode, and J. M. Jordan, "A systematic review of recommendations and guidelines for the management of osteoarthritis: the chronic osteoarthritis management initiative of the U.S. bone and joint initiative," Seminars in Arthritis and Rheumatism, vol. 43, no. 6, pp. 701-712, 2014.

[14] C. Bartholdy, C. Juhl, R. Christensen, H. Lund, W. Zhang, and M. Henriksen, "The role of muscle strengthening in exercise therapy for knee osteoarthritis: a systematic review and metaregression analysis of randomized trials," Seminars in Arthritis and Rheumatism, vol. 47, no. 1, pp. 9-21, 2017.

[15] L. Loew, L. Brosseau, G. P. Kenny et al., "An evidence-based walking program among older people with knee osteoarthritis: the PEP (participant exercise preference) pilot randomized controlled trial," Clinical Rheumatology, vol. 36, no. 7, pp. 1607-1616, 2017.

[16] K. L. Bennell, F. Dobson, and R. S. Hinman, "Exercise in osteoarthritis: moving from prescription to adherence," Best Practice \& Research Clinical Rheumatology, vol. 28, no. 1, pp. 93-117, 2014.

[17] K. A. Scopaz, S. R. Piva, A. B. Gil, J. D. Woollard, C. V. Oddis, and G. K. Fitzgerald, "Effect of baseline quadriceps activation on changes in quadriceps strength after exercise therapy in subjects with knee osteoarthritis," Arthritis \& Rheumatism, vol. 61, no. 7, pp. 951-957, 2009.

[18] M. T. Rafiq, M. S. A. Hamid, and E. Hafiz, "Non-pharmacological interventions for treating symptoms of knee osteoarthritis in overweight or obese patients; a review," Journal of Postgraduate Medical Institute, vol. 34, no. 3, pp. 142-148, 2020.

[19] R. Seguin and M. E. Nelson, "The benefits of strength training for older adults," American Journal of Preventive Medicine, vol. 25, no. 3, pp. 141-149, 2003.

[20] J. H. Kellgren and J. S. Lawrence, "Radiological assessment of osteo-arthrosis," Annals of the Rheumatic Diseases, vol. 16, no. 4, pp. 494-502, 1957.

[21] E. C. Who, "Appropriate body-mass index for Asian populations and its implications for policy and intervention strategies," Lancet (London, England), vol. 363, no. 9403, p. 157, 2004.

[22] M. T. Rafiq, M. S. Abdul Hamid, and E. Hafiz, "The effect of rehabilitation protocol using mobile health in overweight and obese patients with knee osteoarthritis: a clinical trial," Advances in Rheumatology, vol. 61, no. 1, p. 63, 2021.

[23] S. K. Powers and E. T. Howley, Exercise Physiology: Theory and Application to Fitness and Performance, McGraw-Hill, New York, NY, USA, 2007.

[24] W. E. Prentice, "The thigh, hip, groin, and pelvis," Arnheim's Principles of Athletic Training: A Competency-Based Approach, pp. 656-698, NY McGraw Hill, New York, NY, USA, 2003.

[25] M. P. McHugh and M. Nesse, "Effect of stretching on strength loss and pain after eccentric exercise," Medicine \& Science in Sports \& Exercise, vol. 40, no. 3, pp. 566-573, 2008.

[26] M. T. Rafiq, M. S. Hamid, H. A. Eliza, and A. Sakib, "Rehabilitation protocol with or without mobile health in overweight and obese knee osteoarthritis patients-a pilot study," Balneo Research Journal, vol. 10, no. 4, pp. 580-584, 2019.

[27] T. d. S. Alexandre, R. C. Cordeiro, and L. R. Ramos, "Factors associated to quality of life in active elderly," Revista de Saúde Pública, vol. 43, no. 4, pp. 613-621, 2009.

[28] N. Henschke, L. Wouda, C. G. Maher, J. M. Hush, and M. W. van Tulder, "Determinants of patient satisfaction 1 year after presenting to primary care with acute low back pain," The Clinical Journal of Pain, vol. 29, no. 6, pp. 512-517, 2013.

[29] A. Williamson and B. Hoggart, "Pain: a review of three commonly used pain rating scales," Journal of Clinical Nursing, vol. 14, no. 7, pp. 798-804, 2005.

[30] M. J. Hjermstad, P. M. Fayers, D. F. Haugen et al., "Studies comparing numerical rating scales, verbal rating scales, and visual analogue scales for assessment of pain intensity in adults: a systematic literature review," Journal of Pain and Symptom Management, vol. 41, no. 6, pp. 1073-1093, 2011.

[31] G. Jamtvedt, K. T. Dahm, A. Christie et al., "Physical therapy interventions for patients with osteoarthritis of the knee: an overview of systematic reviews," Physical Therapy, vol. 88, no. 1, pp. 123-136, 2008.

[32] C. M. Jenkinson, M. Doherty, A. J. Avery et al., "Effects of dietary intervention and quadriceps strengthening exercises on pain and function in overweight people with knee pain: randomised controlled trial," BMJ, vol. 339, p. b3170, 2009.

[33] K. M. O’Brien, J. Wiggers, A. Williams et al., "Telephonebased weight loss support for patients with knee osteoarthritis: a pragmatic randomised controlled trial," Osteoarthritis and Cartilage, vol. 26, no. 4, pp. 485-494, 2018.

[34] M. T. Rafiq, M. S. Hamid, and E. Hafiz, "Effect of progressive resistance strength training on body mass index, quality of life and functional capacity in knee osteoarthritis: a randomized controlled trial," Journal of Multidisciplinary Healthcare, vol. 14, pp. 2161-2168, 2021.

[35] E. I. Germanou, A. Chatzinikolaou, P. Malliou et al., "Oxidative stress and inflammatory responses following an acute bout of isokinetic exercise in obese women with knee osteoarthritis," The Knee, vol. 20, no. 6, pp. 581-590, 2013.

[36] S. P. Messier, R. F. Loeser, G. D. Miller et al., "Exercise and dietary weight loss in overweight and obese older adults with knee osteoarthritis: the arthritis, diet, and activity promotion trial," Arthritis \& Rheumatism, vol. 50, no. 5, pp. 1501-1510, 2004.

[37] T. E. Lambert, L. A. Harvey, C. Avdalis et al., “An app with remote support achieves better adherence to home exercise programs than paper handouts in people with musculoskeletal conditions: a randomised trial," Journal of Physiotherapy, vol. 63, no. 3, pp. 161-167, 2017.

[38] "Short-term effects of strengthening exercises of lower limb rehabilitation protocol on pain, stiffness, physical function and body mass index among knee osteoarthritis participants who were overweight or obese: a clinical trial," https://www. medrxiv.org/content/10.1101/2021.11.20.21266300v1. 